hep-th/0201149

CALT-68-2371

CITUSC/02-002

\title{
The Equality of Solutions in Vacuum String Field Theory
}

\author{
Takuya Okuda \\ California Institute of Technology 452-48, Pasadena, CA 91125 \\ takuya@theory.caltech.edu
}

\begin{abstract}
We analytically prove that the matter solution of vacuum string field theory constructed by Kostelecky and Potting is the matter sliver state. We also give an analytical proof that the ghost solution by Hata and Kawano is the sliver state in the twisted ghost CFT. It is also proved that the candidate state for the tachyon proposed by Hata and Kawano can be identified with the state constructed by Rastelli, Sen and Zwiebach using CFT. Our proofs are based on the techniques recently developed by Okuyama.
\end{abstract}




\section{Introduction}

In the development of vacuum string field theory (VSFT) [1]-8], two basic approaches have been taken, namely, the operator formalism and the CFT formalism. In the operator formalism, computations can be done explicitly and algebraically. The formalism involves, however, infinite dimensional matrices and their determinants, making it necessary to rely on numerical tests using level truncation. On the other hand, the CFT formalism gives a geometrical picture to various aspects of VSFT and often analytical computations are possible, but the techniques are more abstract. With advantages and disadvantages for each method present, both approaches have been employed to deal with questions in VSFT.

Consider, for example, the equation of motion

$$
\mathcal{Q} \Psi+\Psi * \Psi=0
$$

of VSFT. Since the kinetic operator $\mathcal{Q}$ consists of ghost operators only, for a state of the form $\Psi^{(m)} \otimes \Psi^{(g)}$, the equation of motion can be separated into the matter part and the ghost part:

$$
\Psi^{(m)} * \Psi^{(m)}=\Psi^{(m)}, \mathcal{Q} \Psi^{(g)}+\Psi^{(g)} * \Psi^{(g)}=0
$$

For the matter part, Kostelecky and Potting constructed a solution $\Psi_{m}$ in the operator language 9]. Rastelli, Sen and Zwiebach suggested [3] that this solution can be identified with the matter sliver state $\Xi_{m}$ which is defined as a surface state in the matter CFT, giving numerical evidence based on level truncation.

To have a solution for the ghost part of the equation of motion, the kinetic operator $\mathcal{Q}$ must be fixed. Hata and Kawano showed that on some reasonable assumptions, the existence of a nontrivial solution in the Siegel gauge determines the kinetic operator $\mathcal{Q}$. They then constructed a solution $\Psi_{g}$ and its corresponding kinetic operator in the operator formalism [8]. Gaiotto, Rastelli, Sen, and Zwiebach proposed [10] [11] that the kinetic operator $\mathcal{Q}$ is a mid-point $c$ ghost insertion, and gave a ghost solution for that $\mathcal{Q}$ as the sliver state $\Xi_{g}^{\prime}$ in the twisted ghost CFT. They further proposed that their ghost operator and solution can be identified with those of Hata and Kawano and confirmed this identification by level truncation analysis.

Following the spectrum analysis of the star algebra by Rastelli, Sen, and Zwiebach [12, Okuyama developed methods [13] to analytically prove the equality of the kinetic operators proposed by Hata and Kawano and that defined by Gaiotto, Rastelli, Sen, and Zwiebach.

In this paper, we will use Okuyama's methods to analytically prove the equality of the solution in the operator formalism and that in the CFT formalism. In other words, 
we will analytically show that the matter solution $\Psi_{m}$ by Kostelecky and Potting is the matter sliver state $\Xi_{m}$ and also that the ghost solution $\Psi_{g}$ by Hata and Kawano is the sliver state $\Xi_{g}^{\prime}$ of the twisted ghost CFT. Also, it is proved that the candidate tachyon state proposed by Hata and Kawano is correctly reproduced by the CFT construction as claimed by Rastelli, Sen, and Zwiebach [1].

The outline of this paper is the following. In section 2, we review the methods of Okuyama [13] 14. In section 3, we give analytical proofs that the solutions and the candidate tachyon states in the operator and CFT formalisms are identical.

\section{Review of Methods}

In this section, we review Okuyama's methods [13 and set conventions. The zero momentum sector of the 3-string vertex is given by [15] [16]

$$
\left|V_{3}\right\rangle=\exp \left(\sum_{r, s=1}^{3} \sum_{n, m=1}^{\infty}\left[-\frac{1}{2} a_{n \mu}^{(r) \dagger} V_{n m}^{r s} a_{m}^{(s) \mu \dagger}+c_{-n}^{(r)} \widetilde{V}_{n m}^{r s} b_{-m}^{(s)}+c_{-n}^{(r)} \widetilde{v}_{n}^{r s} b_{0}^{(s)}\right]\right) \bigotimes_{r=1}^{3} c_{0}^{(r)} c_{1}^{(r)}|0\rangle_{r}
$$

We are using the convention $\alpha^{\prime}=1$. We write $M=C V^{11}$, where $C_{n m}=(-1)^{n} \delta_{n m}$ is the twist matrix. In [12, the eigenvectors and the eigenvalues of $M$ were identified. The eigenvector $v^{(k)}$ with eigenvalue $k \in \mathbf{R}$ is defined by the generating function

$$
f_{k}(z)=\sum_{n=1}^{\infty} \frac{v_{n}^{(k)}}{\sqrt{n}} z^{n}=\frac{1}{k}\left(1-e^{-k \tan ^{-1}(z)}\right)
$$

with $f_{k=0}(z)=\lim _{k \neq 0, k \rightarrow 0} f_{k}(z)$.

We introduce the notation

$$
|z\rangle=\left(z, z^{2}, z^{3}, \cdots\right)^{T},|k\rangle=\left(v_{1}^{(k)}, v_{2}^{(k)}, v_{3}^{(k)}, \cdots\right)^{T} .
$$

$\langle z|=| z\rangle^{T}$ is the transpose of $\langle z|$, not the hermitian conjugate. In this notation, the generating function for the eigenvector $|k\rangle$ can be written as

$$
f_{k}(z)=\left\langle z\left|E^{-1}\right| k\right\rangle=\left\langle k\left|E^{-1}\right| z\right\rangle
$$

where $E_{n m}=\sqrt{n} \delta_{n m}$. The crucial properties of $|k\rangle$ are 13]

$$
\begin{aligned}
& \langle k \mid p\rangle=\mathcal{N}(k) \delta(k-p), \\
& \mathbf{1}=\int_{-\infty}^{\infty} d k \mathcal{N}(k)^{-1}|k\rangle\langle k|,
\end{aligned}
$$

where

$$
\mathcal{N}(k)=\frac{2}{k} \sinh \left(\frac{\pi}{2} k\right)
$$


The twist acts as

$$
C|z\rangle=|-z\rangle .
$$

There is a useful relation

$$
z \partial_{z}\langle z|=\langle z| E^{2},
$$

which leads to

$$
\langle z|E| k\rangle=\langle k|E| z\rangle=\frac{z}{1+z^{2}} e^{-k \tan ^{-1}(z)} .
$$

The matter solution by Kostelecky and Potting is [9]

$$
\left|\Psi_{m}\right\rangle=\exp \left(-\frac{1}{2} \sum_{n, m=1}^{\infty} a_{n \mu}^{\dagger} S_{n m} a_{m}^{\mu \dagger}\right)|0\rangle .
$$

The matrix $T \equiv C S$ is given by 9

$$
T=\frac{1}{2 M}(1+M-\sqrt{(1-M)(1+3 M)}) .
$$

The eigenvalues of $M$ and $T$ for the eigenvector $|k\rangle$ are known:

$$
\begin{aligned}
& M(k)=-\frac{1}{1+2 \cosh \left(\frac{\pi}{2} k\right)}, \\
& T(k)=-e^{-\frac{\pi}{2}|k|} .
\end{aligned}
$$

\section{Analytical Proofs}

\subsection{Ghost Solution}

It is convenient to begin with the ghost solution as we will see later. The ghost solution by Hata and Kawano is given as [ [ ]

$$
\left|\Psi_{g}\right\rangle=\exp \left(\sum_{n, m=1}^{\infty} c_{-n} \widetilde{S}_{n m} b_{-m}\right) c_{1}|0\rangle,
$$

where $\widetilde{S}$ can be written in terms of $T[13$ :

$$
\widetilde{S}=-C E T E^{-1} .
$$

On the other hand, the sliver in the twisted ghost CFT is defined by the correlation function 10.

$$
\left\langle\Xi_{g}^{\prime} \mid \phi\right\rangle \equiv\left\langle f \circ \phi^{\prime}(0)\right\rangle^{\prime},
$$


where $\phi$ is an arbitrary Fock state and $f(\xi)=\tan ^{-1}(\xi)$. Since the conformal transformation $f$ acts on the vacuum state as the exponential of a linear combination of the Virasoro generators, we have for the sliver in the twisted ghost CFT,

$$
\left|\Xi_{g}^{\prime}\right\rangle=\exp \left(\sum_{n, m=1}^{\infty} c_{-n} \widehat{S}_{n m} b_{-m}\right)\left|0^{\prime}\right\rangle
$$

The $S L(2, \mathbf{R})$-invariant vacuum $\left|0^{\prime}\right\rangle$ of the twisted CFT is $c_{1}|0\rangle$ of the untwisted CFT. We will prove the equality of the two states by showing that the generating functions for $\widehat{S}$ and $\widetilde{S}$ are the same.

To compute the generating function $\langle z|\widetilde{S}| w\rangle$, let us consider the function [10]

$$
\begin{aligned}
h(z, w) & \equiv\left\langle\Xi_{g}^{\prime}\left|R\left(c^{\prime}(w) b^{\prime}(z)\right) c_{0}\right| 0^{\prime}\right\rangle \\
& =\left\langle 0^{\prime}\left|\exp \left(-\sum_{n, m=1}^{\infty}(-1)^{n+m} c_{n} \widehat{S}_{n m} b_{m}\right) R\left(c^{\prime}(w) b^{\prime}(z)\right) c_{0}\right| 0^{\prime}\right\rangle \\
& =\left\langle f \circ c^{\prime}(w) f \circ b^{\prime}(z) f \circ c^{\prime}(0)\right\rangle^{\prime} .
\end{aligned}
$$

Here $R$ denotes radial ordering and the extra signs in (3.5) come from BPZ conjugation. The expression (3.5) allows us to write this function as ${ }^{1}$

$$
h(z, w)=\sum_{n, m=1}^{\infty}(-w)^{m}(-z)^{n-1} \widehat{S}_{n m}+\frac{w}{z(w-z)} .
$$

Another expression for $h(z, w)$ can be obtained using (3.6]):

$$
\begin{aligned}
h(z, w) & =\left\langle c^{\prime}(f(w)) b^{\prime}(f(z)) \frac{d f(z)}{d z} c^{\prime}(f(0))\right\rangle^{\prime} \\
& =\frac{1}{1+z^{2}} \frac{1}{\tan ^{-1}(w)-\tan ^{-1}(z)} \frac{\tan ^{-1}(w)}{\tan ^{-1}(z)} .
\end{aligned}
$$

Note that the $c$ ghost has weight zero and the $b$ ghost has weight one in the twisted ghost CFT. Combining eqs.(3.7) and (3.8), we obtain the generating function of $\widehat{S}$.

$$
\begin{aligned}
\langle-z|\widehat{S}|-w\rangle & =\frac{z}{1+z^{2}} \frac{1}{\tan ^{-1}(z)-\tan ^{-1}(w)} \frac{\tan ^{-1}(w)}{\tan ^{-1}(z)}+\frac{w}{w-z} \\
& =\langle z|\widehat{S}| w\rangle .
\end{aligned}
$$

Now we compute $\langle z|\widetilde{S}| w\rangle$. Because of the twist matrix $C$ in the definition of $S$, it is simpler to use $-z$ instead of $z$.

$$
\langle-z|\widetilde{S}| w\rangle=-\int_{-\infty}^{\infty} d k\langle z|E T| k\rangle N(k)^{-1}\left\langle k\left|E^{-1}\right| w\right\rangle
$$

\footnotetext{
${ }^{1}$ In [10], the second term of (3.7) was missing. This term arises from contraction of $c^{\prime}(w), b^{\prime}(z)$, and $c_{0}$ and is identical with $\left\langle c^{\prime}(w) b^{\prime}(z) c^{\prime}(0)\right\rangle^{\prime}$.
} 


$$
\begin{aligned}
= & -\int_{-\infty}^{\infty} d k \frac{k}{2} \frac{1}{\sinh \left(\frac{\pi}{2} k\right)}\left(-e^{-\frac{\pi}{2}|k|}\right) \frac{z}{1+z^{2}} e^{-k \tan ^{-1}(z)} \\
& \times \frac{1}{k}\left(1-e^{-k \tan ^{-1}(w)}\right) \\
= & \frac{z}{1+z^{2}} \int_{0}^{\infty} d k \frac{e^{-\frac{\pi}{2}|k|}}{\sinh \left(\frac{\pi}{2} k\right)}(\sinh ((\theta+\phi) k)-\sinh (\theta k))
\end{aligned}
$$

Here $\theta \equiv \tan ^{-1}(z), \phi \equiv \tan ^{-1}(w)$, and we used eqs. (2.6), (2.7), (2.10), and (2.14). We now use the formula

$$
\int_{0}^{\infty} d k e^{-\frac{\pi}{2} k} \frac{\sinh (\theta k)}{\sinh \left(\frac{\pi}{2} k\right)}=\frac{1}{\theta}-\frac{1}{\tan \theta}, \quad(|\operatorname{Re} \theta|<\pi)
$$

to obtain

$$
\langle-z|\widetilde{S}| w\rangle=-\frac{z}{1+z^{2}} \frac{\tan ^{-1}(w)}{\tan ^{-1}(z)\left(\tan ^{-1}(z)+\tan ^{-1}(w)\right)}+\frac{w}{z+w} .
$$

If we replace $z$ by $-z$, this expression exactly coincides with eq.(3.9). This completes the proof of the equality $\Psi_{g}=\Xi_{g}^{\prime}$.

\subsection{Matter Solution}

The solution by Kostelecky and Potting is given in the operator language [9]:

$$
\left|\Psi_{m}\right\rangle=\exp \left(-\frac{1}{2} \sum_{n, m=1}^{\infty} a_{n \mu}^{\dagger} S_{n m} a_{m}^{\mu \dagger}\right)|0\rangle
$$

We have for the matter sliver 3 ]

$$
\left|\Xi_{m}\right\rangle=\exp \left(-\frac{1}{2} \sum_{n, m=1}^{\infty} a_{n \mu}^{\dagger} S_{n m}^{\prime} a_{m}^{\mu \dagger}\right)|0\rangle,
$$

with the coefficient matrix $S^{\prime}$ determined by the defining equation [5]

$$
\left\langle\Xi_{m} \mid \phi\right\rangle \equiv\langle f \circ \phi(0)\rangle,
$$

where $\phi$ is any Fock state and $f(\xi)=\tan ^{-1}(\xi)$. Proceeding in the same way as for the ghost solution, we will prove the equality of these two states by computing the generating functions of $S$ and $S^{\prime}$.

To obtain the generating function of $S^{\prime}$, consider the tensor function

$$
\begin{aligned}
h^{\mu \nu}(z, w) & \equiv\left\langle\Xi_{m}\left|R\left(\partial X^{\mu}(w) \partial X^{\nu}(z)\right)\right| 0\right\rangle \\
& =\left\langle 0\left|\exp \left(-\frac{1}{2} \sum_{n, m=1}^{\infty}(-1)^{n+m} a_{n \mu}^{\dagger} S_{n m}^{\prime} a_{m}^{\mu \dagger}\right) R\left(\partial X^{\mu}(w) \partial X^{\nu}(z)\right)\right| 0\right\rangle \\
& =\left\langle f \circ \partial X^{\mu}(w) f \circ \partial X^{\nu}(z)\right\rangle
\end{aligned}
$$


The signs in (3.16) arise because of BPZ conjugation. From the expression (3.16), we get

$$
h^{\mu \nu}(z, w)=\frac{1}{2} \sum_{n, m=1}^{\infty}(-w)^{n-1}(-z)^{m-1} \sqrt{n m} S^{\prime n m} \eta^{\mu \nu}-\frac{1}{2} \eta^{\mu \nu} \frac{1}{(z-w)^{2}} .
$$

The other expression (3.17) allows us to compute $h^{\mu \nu}(z, w)$ as

$$
\begin{aligned}
h^{\mu \nu}(z, w) & =\frac{d f(w)}{d w} \frac{d f(z)}{d z}\left\langle\partial X^{\mu}(f(w)) \partial X^{\nu}(f(z))\right\rangle \\
& =\frac{1}{1+w^{2}} \frac{1}{1+z^{2}}\left(-\frac{1}{2}\right) \eta^{\mu \nu} \frac{1}{\left(\tan ^{-1}(z)-\tan ^{-1}(w)\right)^{2}} .
\end{aligned}
$$

Comparing eqs.(3.18) and (3.19) gives the generating function

$$
\begin{aligned}
\left\langle-z\left|E S^{\prime} E\right|-w\right\rangle & =\frac{z w}{(z-w)^{2}}-\frac{z w}{\left(1+z^{2}\right)\left(1+w^{2}\right)} \frac{1}{\left(\tan ^{-1}(z)-\tan ^{-1}(w)\right)^{2}} \\
& =\left\langle z\left|E S^{\prime} E\right| w\right\rangle .
\end{aligned}
$$

The generating function $\langle z|E S E| w\rangle$ for $S$ can be computed from the generating function for the ghost solution.

$$
\begin{aligned}
\langle-z|E S E| w\rangle & =\langle z|E T E| w\rangle \\
& =w \frac{\partial}{\partial w}\left\langle z\left|E T E^{-1}\right| w\right\rangle \\
& =-w \frac{\partial}{\partial w}\langle-z|\widetilde{S}| w\rangle \\
& =-w \frac{\partial}{\partial w}\left(-\frac{z}{1+z^{2}} \frac{\tan ^{-1}(w)}{\tan ^{-1}(z)\left(\tan ^{-1}(z)+\tan ^{-1}(w)\right)}+\frac{w}{z+w}\right) \\
& =\frac{z w}{\left(1+z^{2}\right)\left(1+w^{2}\right)} \frac{1}{\left(\tan ^{-1}(z)+\tan ^{-1}(w)\right)^{2}}-\frac{z w}{(z+w)^{2}} .
\end{aligned}
$$

Here we used eqs. (2.9), (3.2), and (3.12). Recovering the normal sign, we see that eq.(3.21) precisely agrees with eq.(3.20). This shows $S=S^{\prime}$ and completes the proof for the matter solution.

\subsection{Candidate State for the tachyon}

The Hata-Kawano state, which is a candidate state for the tachyon, is given by [8]

$$
\exp \left(-\frac{1}{2} \sum_{n, m=1}^{\infty} a_{n}^{\dagger} S_{n m} a_{m}^{\dagger}-a_{0} \sum_{n=1}^{\infty} t_{n} a_{n}^{\dagger}\right)|k\rangle
$$


where

$$
\left(t_{n}\right)=|t\rangle=-\frac{(1-T)^{2}}{(1-M)(1+T)}\left|v_{+0}+v_{-0}\right\rangle .
$$

The vector $v_{+0}+v_{-0}$ is related to $v_{e}$ which was introduced in [14 through

$$
\left|v_{+0}+v_{-0}\right\rangle=2\left|v_{e}\right\rangle
$$

In [14, it was found that

$$
\left\langle k \mid v_{e}\right\rangle=\frac{1}{k} \frac{\cosh \left(\frac{\pi k}{2}\right)-1}{2 \cosh \left(\frac{\pi k}{2}\right)+1} .
$$

The matrix factor in (3.23) can also be simplified:

$$
\frac{(1-T)^{2}}{(1-M)(1+T)}=\frac{T^{2}-T+1}{1+T} .
$$

Therefore,

$$
\begin{aligned}
|t\rangle & =-2 \frac{T^{2}-T+1}{1+T} \int_{-\infty}^{\infty} d k|k\rangle \frac{k}{2 \sinh \left(\frac{\pi}{2}\right)} \frac{1}{k} \frac{\cosh \left(\frac{\pi}{2} k\right)-1}{2 \cosh \left(\frac{\pi}{2} k\right)+1} \\
& =-\frac{1}{2} \int_{-\infty}^{\infty} d k|k\rangle \frac{T^{2}(k)-T(k)+1}{1+T(k)} \frac{(1+T(k))^{2}}{T^{2}(k)-T(k)+1} \frac{1}{\sinh \left(\frac{\pi}{2} k\right)} \\
& =-\frac{1}{2} \int_{-\infty}^{\infty} d k|k\rangle \frac{\left(1-e^{-\frac{\pi}{2}|k|}\right)}{\sinh \left(\frac{\pi}{2} k\right)} .
\end{aligned}
$$

Now let us consider the generating function $\langle z|E| t\rangle$.

$$
\begin{aligned}
\langle z|E| t\rangle & =-\frac{1}{2} \int_{-\infty}^{\infty} d k \frac{z}{1+z^{2}} e^{-k \tan ^{-1}(z)} \frac{\left(1-e^{-\frac{\pi}{2}|k|}\right)}{\sinh \left(\frac{\pi}{2} k\right)} \\
& =\frac{z}{1+z^{2}} \int_{0}^{\infty} d k\left(1-e^{-\frac{\pi}{2} k}\right) \frac{\sinh (\theta k)}{\sinh \left(\frac{\pi}{2} k\right)}
\end{aligned}
$$

Here, $\theta=\tan ^{-1}(z)$. Using the formula (3.11) and

$$
\int_{0}^{\infty} d k \frac{\sinh (\theta k)}{\sinh \left(\frac{\pi}{2} k\right)}=\tan \theta,\left(|\operatorname{Re} \theta|<\frac{\pi}{2}\right),
$$

we obtain

$$
\langle z|E| t\rangle=1-\frac{z}{1+z^{2}} \frac{1}{\tan ^{-1}(z)} .
$$

Rastelli, Sen, and Zwiebach proposed that the Hata-Kawano state is given by

$$
\left\langle\chi_{T}(k) \mid \psi\right\rangle \equiv \mathcal{N} \lim _{n \rightarrow \infty} n^{2 k^{2}}\left\langle e^{i k \cdot X\left(\frac{n \pi}{4}\right)} f \circ \psi(0)\right\rangle_{C_{n}},
$$


where $\mathcal{N}$ is a normalization constant and $C_{n}$ is the upper half plane with the identification $z \simeq z+\frac{n}{2} \pi[5]$. Here we will prove their proposal. The insertion of the tachyon vertex operator produces a linear term in the exponential:

$$
\left|\chi_{T}(k)\right\rangle=\exp \left(-\frac{1}{2} \sum_{n, m=1}^{\infty} a_{n}^{\dagger} S_{n m}^{\prime} a_{m}^{\dagger}-a_{0} \sum_{n=1}^{\infty} \widehat{t}_{n} a_{n}^{\dagger}\right)|k\rangle
$$

The coefficient matrix $S^{\prime}$ is the one for the matter sliver, for the quadratic term in the exponential represents the action of the same conformal transformation $f$ on the vacuum. This can also be directly checked by computing a two-point correlation function. To compute the coefficient vector $\hat{t}$, let us consider the function

$$
\begin{aligned}
\left\langle\chi_{T}(k)\left|\partial X^{\mu}(z)\right| k^{\prime}\right\rangle & =\left\langle k\left|\exp \left(-\frac{1}{2} a \cdot S^{\prime} \cdot a-a_{0} \hat{t} \cdot C \cdot a\right) \partial X^{\mu}(z)\right| k^{\prime}\right\rangle \\
& =-i k^{\prime \mu}\left\langle k \mid k^{\prime}\right\rangle \frac{1}{z}\left(1-\sum_{m=1}^{\infty}(-1)^{m} \sqrt{m} z^{m} \widehat{t}^{m}\right) .
\end{aligned}
$$

Here we have used

$$
\begin{aligned}
& \partial X^{\mu}(z)=\frac{p^{\mu}}{i z}+\frac{1}{\sqrt{2} i} \sum_{m \neq 0} \frac{\alpha_{m}^{\mu}}{z^{m+1}}, \\
& a_{0}=a_{0}^{\dagger}=\sqrt{2} p .
\end{aligned}
$$

On the other hand, from definition (3.34), we have

$$
\begin{aligned}
\left\langle\chi_{T}(k)\left|\partial X^{\mu}(z)\right| k^{\prime}\right\rangle & =\mathcal{N} \lim _{n \rightarrow \infty} n^{2 k^{2}}\left\langle e^{i k \cdot X\left(\frac{n \pi}{4}\right)} f \circ \partial X^{\mu}(z) f \circ e^{i k^{\prime} \cdot X(0)}\right\rangle_{C_{n}} \\
& \propto \lim _{w \rightarrow \infty}\left\langle e^{i k \cdot X(w)} f \circ \partial X^{\mu}(z) f \circ e^{i k^{\prime} \cdot X(0)}\right\rangle_{U H P} \\
& \propto \frac{-i k^{\prime \mu}}{1+z^{2}} \frac{1}{\tan ^{-1}(z)}\left\langle k \mid k^{\prime}\right\rangle .
\end{aligned}
$$

By comparing (3.37) and (3.42) and choosing $\mathcal{N}$ so that both have the same residue for the pole at $z=0$, we get

$$
\langle\hat{t}|E|-z\rangle=1-\frac{z}{1+z^{2}} \frac{1}{\tan ^{-1}(z)}=\langle\hat{t}|E| z\rangle,
$$

which is identical to eq. (3.33). This completes the proof for the candidate tachyon state.

\section{Acknowledgments}

I would like to thank Yuji Okawa and Hirosi Ooguri for valuable discussions. 


\section{References}

[1] L. Rastelli, A. Sen and B. Zwiebach, "String field theory around the tachyon vacuum," arXiv:hep-th/0012251.

[2] L. Rastelli, A. Sen and B. Zwiebach, "Vacuum string field theory," arXiv:hep-th/0106010.

[3] L. Rastelli, A. Sen and B. Zwiebach, "Classical solutions in string field theory around the tachyon vacuum," arXiv:hep-th/0102112.

[4] L. Rastelli, A. Sen and B. Zwiebach, "Half strings, projectors, and multiple D-branes in vacuum string field theory," JHEP 0111, 035 (2001) arXiv:hep-th/0105058.

[5] L. Rastelli, A. Sen and B. Zwiebach, "Boundary CFT construction of D-branes in vacuum string field theory," JHEP 0111, 045 (2001) arXiv:hep-th/0105168.

[6] D. J. Gross and W. Taylor, "Split string field theory. I," JHEP 0108, 009 (2001) arXiv:hep-th/0105059.

[7] D. J. Gross and W. Taylor, "Split string field theory. II," JHEP 0108, 010 (2001) arXiv:hep-th/0106036.

[8] H. Hata and T. Kawano, "Open string states around a classical solution in vacuum string field theory," JHEP 0111, 038 (2001) arXiv:hep-th/0108150.

[9] V. A. Kostelecky and R. Potting, "Analytical construction of a nonperturbative vacuum for the open bosonic string," Phys. Rev. D 63, 046007 (2001) arXiv:hep-th/0008252.

[10] D. Gaiotto, L. Rastelli, A. Sen and B. Zwiebach, "Ghost structure and closed strings in vacuum string field theory," arXiv:hep-th/0111129.

[11] L. Rastelli, A. Sen and B. Zwiebach, "A note on a proposal for the tachyon state in vacuum string field theory," arXiv:hep-th/0111153

[12] L. Rastelli, A. Sen and B. Zwiebach, "Star algebra spectroscopy," arXiv:hep-th/0111281.

[13] K. Okuyama, "Ghost kinetic operator of vacuum string field theory," arXiv:hep-th/0201015.

[14] K. Okuyama, "Ratio of tensions from vacuum string field theory," arXiv:hep-th/0201136. 
[15] D. J. Gross and A. Jevicki, "Operator Formulation Of Interacting String Field Theory," Nucl. Phys. B 283, 1 (1987).

[16] D. J. Gross and A. Jevicki, "Operator Formulation Of Interacting String Field Theory. 2," Nucl. Phys. B 287, 225 (1987). 